\title{
Three-Dimensional Model of Magnetic Reluctance of Rectangular Iron Core"
}

\author{
Satoru FUKATA ${ }^{* *}$ \\ ${ }^{* *}$ Faculty of Design, Kyushu University, \\ 4-9-1 Shiobaru, Mimamiku, Fukuoka, 815-8540, Japan \\ E-mail: fukata@design.kyushu-u.ac.jp
}

\begin{abstract}
This study examines a three-dimensional (3D) distribution of a magnetic field to obtain an analytical model of magnetic reluctance of rectangular iron cores whose ends face a working air gap in a magnetic system. An analytical solution is obtained without the boundary condition that the magnetic intensity is uniform in the direction of a magnetomotive force at the edge of an iron core's cross-section. Furthermore, the theory of generalized function (or distribution) is applied to convergence of a trigonometric infinite series. Consequently, the solution is an approximation. The magnetic reluctance is obtained from the ratio of the magnetomotive force along the iron-core surface to the magnetic flux. The reluctance is expressed with a part corresponding to a $2 \mathrm{D}$ model and a part with ends effect. To assess the model, approximated equations to simplify the calculation are given with numerical examples to compare the model with a 2D model without ends effect.
\end{abstract}

Key words: Actuator, Magnetic Actuator, Modeling, Magnetic Reluctance, Rectangular Iron Core, Frequency Characteristics

\section{Introduction}

Solid iron cores are used in electromagnetic actuators in difficult cases for laminated iron cores or to achieve some objective. For a simple composition of the actuators, it is expedient to apply the magnetic circuit theory to the analysis and design. The magnetic reluctance plays an important role. A simple two-dimensional (2D) model was presented for rectangular iron cores whose ends face an air gap. ${ }^{(1),(2)}$ This model neglected the field distribution near the ends where magnetic flux enters and exits: the so-called ends effect. This model is useful in practical applications also in another shape of I or U; it is good for lower frequencies, but becomes poorer with increasing frequency.(2), (3) The error is attributable to a lack of reasonable conditions in the modeling. This paper describes an attempt at deriving a 3D model with the ends effect to check the usefulness of the 2D model.

We assume that the magnetic constants are uniform and constant, and that the field distribution is uniform on and perpendicular to the end surfaces facing the air gap. These assumptions are necessary to derive an analytical solution, but the former is not true in general and the latter is considered to be only roughly valid. We will neglect a boundary condition by which the magnetic field in the direction of magnetomotive force is uniform at the edge of a cross-section of an iron core. We apply the theory of generalized function (or distribution) to discussion of the convergence of a trigonometric infinite series. Therefore,

*Received 25 Nov., 2008 (No. T1-06-0250) Japanese Original : Trans. Jpn. Soc. Mech. Eng., Vol.73, No.725, C (2007), pp.192-198 (Received 16 Mar., 2006) [DOI: 10.1299/jee.3.444] 
the solution obtained is an approximation. Magnetic reluctance is modeled from the ratio of the magnetomotive force to the total flux into the iron core.

In general, numerical analysis is useful for such a problem. In contrast, the author used analytical methods in modeling of infinitely wide iron cores. ${ }^{(4)}$ In addition, a similar technique is used to apply a result in the preceding modeling and to compare the two models in a similar form.

\section{Notations and Symbols}

$\begin{array}{ll}a: & \text { Width of the rectangular core } \\ b: & \text { Height (Thickness) of the rectangular core } \\ H_{0}: & \text { Uniform magnetic intensity on the pole faces } \\ H_{x}, H_{y}, H_{z}: & \text { Magnetic intensity } \\ h_{x}, h_{y}, h_{z}: & \text { Normalized magnetic intensity with Laplace transform } \\ j=\sqrt{-1}: & \text { Imaginary unit } \\ l: & \text { Length of iron core } \\ p_{a n}, p_{b n}: & \text { Parameters } p_{a n}=\frac{2 n \pi}{a}, p_{b n}=\frac{2 n \pi}{b}\end{array}$

(Correspondence between suffixes and letters is similar below.)

$\begin{array}{ll}q_{a m}, q_{b m}: & \text { Parameters, } q_{a m}=\frac{(2 m+1) \pi}{a} \\ r_{m}: & \text { Relative magnetic reluctance of the 3D model } \\ r_{m a b}: & \text { Part without core length of } r_{m} \\ r_{m a p}: & \text { Approximation of } r_{m} \\ r_{m 2}: & \text { Relative magnetic reluctance of the 2D model } \\ r_{m \infty}: & \text { Approximation at higher frequencies of } r_{m} \\ s: & \text { Variables of Laplace transform } \\ \alpha^{2}=\mu \sigma s & \\ \beta_{a n}, \beta_{b n}: & \text { Parameters, } \beta_{a n}{ }^{2}=\alpha^{2}+p_{a n}{ }^{2} \\ \gamma_{a m}, \gamma_{b m}: & \text { Parameters, } \gamma_{a m}{ }^{2}=\alpha^{2}+q_{a m}{ }^{2} \\ \mu: & \text { Permeability of iron core } \\ \sigma: & \text { Inductivity of iron core }\end{array}$

\section{Problem Statement and Assumptions of Analysis}

We consider a rectangular iron core of width $a$, height (thickness) $b$ and length $l$, whose ends face an air gap as shown in Fig.1. Magnetic flux enters the end on the left-hand side, passing through the iron core and goes out of the other end. The problem is to obtain an analytical model of the magnetic reluctance in this case.

For simplicity, we make the following assumptions.

(1) Magnetic constants are uniform and constant (no magnetic saturation).

(2) The magnetic field is uniform on and is normal to the end surfaces.

(3) No flux leakage.

(4) The boundary condition is neglected that the magnetic field is uniform at the edge of a cross-section of the iron core. 


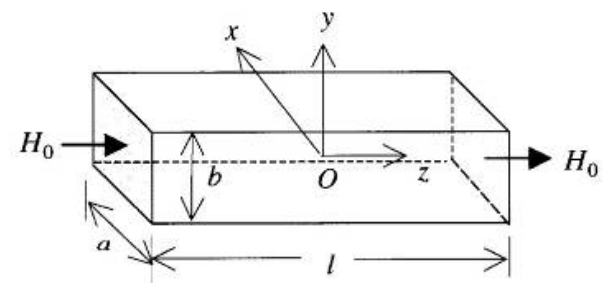

Fig. 1 Iron core and coordinate system

\section{Distribution of Magnetic Intensity}

We address the coordinate system presented in Fig. 1: the origin is at the center of the core, the axes $x, y$ and $z$ are in the directions of width, height and length, respectively.

The components of magnetic intensity, $H_{x}, H_{y}$, and $H_{z}$, in the three axes are presumed to have the distributions of the form

$$
\begin{aligned}
& H_{x} \text { is odd for } x \text {, even for } y \text { and odd for } z \text {; } \\
& H_{y} \text { is even for } x \text { and odd for } y \text { and } z \text {; and } \\
& H_{z} \text { is even for } x, y \text { and } z .
\end{aligned}
$$

Those field distributions are functions of time $t$; but its description will be omitted for simplicity.

\subsection{Magnetic Field Equations}

For a homogeneous magnetic material with permeability $\mu$ and conductivity $\sigma$, we have the equations of the fields as follows. ${ }^{(5),(6)}$

$$
\begin{aligned}
& \nabla^{2} H_{x}=\mu \sigma \frac{\partial H_{x}}{\partial t} \\
& \nabla^{2} H_{y}=\mu \sigma \frac{\partial H_{y}}{\partial t} \\
& \nabla^{2} H_{z}=\mu \sigma \frac{\partial H_{z}}{\partial t} \\
& \frac{\partial H_{x}}{\partial x}+\frac{\partial H_{y}}{\partial y}+\frac{\partial H_{z}}{\partial z}=0
\end{aligned}
$$

Therein, $\nabla^{2}$ is the Laplacian defined as

$$
\nabla^{2}=\frac{\partial}{\partial x^{2}}+\frac{\partial}{\partial y^{2}}+\frac{\partial}{\partial z^{2}} .
$$

The boundary conditions are described from Assumptions (2) and (3) as shown below.

$$
\begin{aligned}
& H_{z}\left(x, y, \pm \frac{l}{2}\right)=H_{0} \\
& H_{x}\left( \pm \frac{a}{2}, y, z\right)=0, H_{x}\left(x, \pm \frac{b}{2}, z\right)=0 \\
& H_{y}\left(x, \pm \frac{b}{2}, z\right)=0, H_{y}\left( \pm \frac{a}{2}, y, z\right)=0
\end{aligned}
$$

In those expressions, $H_{0}=H_{0}(t)$ is a uniform intensity on the pole faces. The other 
boundary condition is that the distribution of $H_{z}$ is uniform around the surface of the same position of $z$ :

$$
H_{z}\left( \pm \frac{a}{2}, y, z\right)=H_{z}\left(x, \pm \frac{b}{2}, z\right) .
$$

Another constraint is the continuity of magnetic flux, as

$$
\int_{-b / 2}^{b / 2} \int_{-a / 2}^{a / 2} H_{z}(x, y, z) d x d y=a b H_{0} .
$$

It seems difficult to obtain an analytical solution to satisfy all the boundary conditions, Eqs. (6)-(9). Therefore, we try to solve the equations without the condition of Eq. (9); the obtained solution is an approximation.

\subsection{Equations of Approximated Solution}

We take the Laplace transforms to solve the equations with the initial values of zero. Then, we use the normalized variables, for example, defined as

$$
h_{x}=h_{x}(x, y, z, s)=\frac{H_{x}(x, y, z, s)}{H_{0}(s)},
$$

where $H_{x}(x, y, z, s)$ is the Laplace transform of $H_{x}(x, y, z)=H_{x}(x, y, z, t)$ and $H_{0}(s)$ of $H_{0}=H_{0}(t)$. In this case, Eqs. (1)-(8) and (10) are transformed to the following.

$$
\begin{aligned}
& \nabla^{2} h_{x}=\alpha^{2} h_{x}, \quad \nabla^{2} h_{y}=\alpha^{2} h_{y} \\
& \nabla^{2} h_{z}=\alpha^{2} h_{z} \\
& \frac{\partial h_{x}}{\partial x}+\frac{\partial h_{y}}{\partial y}+\frac{\partial h_{z}}{\partial z}=0
\end{aligned}
$$

Therein, the following hold.

$$
\begin{aligned}
& \alpha^{2}=\mu \sigma s \\
& h_{x}\left( \pm \frac{a}{2}, y, z, s\right)=0, h_{x}\left(x, \pm \frac{b}{2}, z, s\right)=0 \\
& h_{y}\left(x, \pm \frac{b}{2}, z, s\right)=0, h_{y}\left( \pm \frac{a}{2}, y, z, s\right)=0 \\
& h_{z}\left(x, y, \pm \frac{l}{2}, s\right)=1 \\
& \int_{-b / 2}^{b / 2} \int_{-a / 2}^{a / 2} h_{z}(x, y, z, s) d x d y=a b
\end{aligned}
$$

\subsection{Expressions of Magnetic Intensity}

First we solve Eq. (12) using the variable separable method; then, by considering the presumed forms of the fields and the boundary conditions of Eqs. (16) and (17), we obtain the solutions shown below.

$$
\begin{aligned}
& h_{x}=\sum_{n=1}^{\infty} \sum_{m=0}^{\infty} a_{n m} \sin \left(p_{a n} x\right) \cos \left(q_{b m} y\right) \frac{\sinh \left(\xi_{n m} z\right)}{\cosh \left(\xi_{n m} l / 2\right)} \\
& h_{y}=\sum_{n=1}^{\infty} \sum_{m=0}^{\infty} b_{n m} \sin \left(p_{b n} y\right) \cos \left(q_{a m} x\right) \frac{\sinh \left(\eta_{n m} z\right)}{\cosh \left(\eta_{n m} l / 2\right)}
\end{aligned}
$$

In those equations, the following hold.

$$
\begin{aligned}
& \xi_{n m}{ }^{2}=\alpha^{2}+p_{a n}{ }^{2}+q_{b m}{ }^{2}, \quad \eta_{n m}{ }^{2}=\alpha^{2}+p_{b n}{ }^{2}+q_{a m}{ }^{2} \\
& p_{a n}=\frac{2 \pi}{a} n, \quad p_{b n}=\frac{2 \pi}{b} n \\
& q_{a m}=\frac{\pi}{a}(2 m+1), \quad q_{b m}=\frac{\pi}{b}(2 m+1)
\end{aligned}
$$

Regarding $h_{z}$, it is simple to solve Eq. (14) instead of Eq. (13). Then, considering the 
boundary condition of Eq. (18) and taking a similar expression in Ref. (4), we write the solution as

$$
\begin{aligned}
h_{z}=1 & +\sum_{n, m}^{\infty} c_{n m} \cos \left(p_{a n} x\right) \cos \left(q_{b m} y\right)\left[1-\frac{\cosh \left(\xi_{n m} z\right)}{\cosh \left(\xi_{n m} l / 2\right)}\right] \\
& +\sum_{n, m}^{\infty} d_{n m} \cos \left(p_{b n} y\right) \cos \left(q_{a m} x\right)\left[1-\frac{\cosh \left(\eta_{n m} z\right)}{\cosh \left(\eta_{n m} l / 2\right)}\right],
\end{aligned}
$$

where the constants are expected to satisfy the following relations.

$$
a_{n m}=\frac{\xi_{n m}}{p_{a n}} c_{n m}, \quad b_{n m}=\frac{\eta_{n m}}{p_{b n}} d_{n m}
$$

Next, substituting Eq. (25) into Eq. (13) and arranging the result, we have the relation

$$
\nabla^{2} h_{z}=\alpha^{2} h_{z}-\alpha^{2} f(x, y),
$$

where

$$
\begin{aligned}
f(x, y)=1+\frac{1}{\alpha^{2}} \sum_{n, m}^{\infty}[ & c_{n m} \xi_{n m}{ }^{2} \cos \left(p_{a n} x\right) \cos \left(q_{b m} y\right) \\
& \left.+d_{n m} \eta_{n m}{ }^{2} \cos \left(p_{b n} y\right) \cos \left(q_{a m} x\right)\right] .
\end{aligned}
$$

We must determine the constants to satisfy $f(x, y)=0$. However, it seems difficult to do so in a usual way.

Equation (25) is a function of $y$ at $x= \pm a / 2$ and of $x$ at $y= \pm b / 2$, which does not satisfy the boundary condition of Eq. (9). Therefore, the solution presented above is an approximated expression.

To have a generalized form including the infinitely wide iron core $(a \rightarrow \infty, 1 \mathrm{D}$ iron core) in the above expressions, we write the constants as

$$
c_{n m}=\frac{b}{a+b} \bar{c}_{n m}, \quad d_{n m}=\frac{a}{a+b} \bar{d}_{n m} .
$$

Then, Eq. (28) is rewritten as presented below.

$$
\begin{aligned}
f(x, y) & =\frac{b}{a+b}\left[1+\sum_{n, m}^{\infty} \frac{\xi_{n m}{ }^{2}}{\alpha^{2}} \bar{c}_{n m} \cos \left(p_{a n} x\right) \cos \left(q_{b m} y\right)\right] \\
& +\frac{a}{a+b}\left[1+\sum_{n, m}^{\infty} \frac{\eta_{n m}{ }^{2}}{\alpha^{2}} \bar{d}_{n m} \cos \left(p_{b n} y\right) \cos \left(q_{a m} x\right)\right]
\end{aligned}
$$

\section{Determination of Constants with Generalized Function}

\subsection{Fourier Series of Dirac Delta Function}

The Dirac delta function is complicated in general. In the space of generalized function (or distribution), we obtain the following expression with the Fourier series. ${ }^{\text {(7), (8) }}$

$$
\delta_{T}(t) \equiv \sum_{n=-\infty}^{n=\infty} \delta(t-n T)=\frac{1}{T}+\frac{2}{T} \sum_{n=1}^{\infty} \cos \left(\frac{2 \pi n}{T} t\right)
$$

In that equation, $T$ is the period. The infinite series of the above equation does not converge in the conventional function space. From this equation, we have the following series.

$$
\delta_{T}\left(t-\frac{T}{2}\right)=\frac{1}{T}+\frac{2}{T} \sum_{n=1}^{n=\infty}(-1)^{n} \cos \left(\frac{2 \pi n}{T} t\right)
$$

With $T=a$ and $t=x$ for $|x| \leq a / 2$, we obtain the equation shown below.

$$
\delta_{a}(x) \equiv \delta\left(x+\frac{a}{2}\right)+\delta\left(x-\frac{a}{2}\right)=\frac{1}{a}+\frac{2}{a} \sum_{n=1}^{\infty}(-1)^{n} \cos \left(\frac{2 \pi n}{a} x\right)
$$




\subsection{Determination of Constants}

We have the Fourier series expansion of ${ }^{(7)}$

$$
1=\frac{4}{\pi} \sum_{m=0}^{\infty} \frac{(-1)^{m}}{2 m+1} \cos \left[\frac{\pi}{b}(2 m+1) y\right],|y|<\frac{b}{2} .
$$

Multiplying the second term in Eq. (33) by this equation yields

$$
a \delta_{a}(x)=1+\frac{8}{\pi} \sum_{n} \sum_{m} \frac{(-1)^{n+m}}{2 m+1} \cos \left(p_{a n} x\right) \cos \left(q_{b m} y\right) .
$$

Similarly, we obtain the following.

$$
b \delta_{b}(y)=1+\frac{8}{\pi} \sum_{n, m} \frac{(-1)^{n+m}}{2 m+1} \cos \left(p_{b n} y\right) \cos \left(q_{a m} x\right),|x|<\frac{a}{2}, \quad|y| \leq \frac{b}{2}
$$

In Eq. (30) if we set the constants as

$$
\bar{c}_{n m}=\frac{8}{\pi} \frac{(-1)^{n+m}}{2 m+1} \frac{\alpha^{2}}{\xi_{n m}{ }^{2}} \quad \text { and } \quad \bar{d}_{n m}=\frac{8}{\pi} \frac{(-1)^{n+m}}{2 m+1} \frac{\alpha^{2}}{\eta_{n m}{ }^{2}},
$$

then we have the following relation.

$$
f(x, y)=\frac{a b}{a+b}\left[\delta\left(x+\frac{a}{2}\right)+\delta\left(x-\frac{a}{2}\right)+\delta\left(y+\frac{b}{2}\right)+\delta\left(y-\frac{b}{2}\right)\right]
$$

Therefore, $f(x, y)=0$ in Eq. (27) except at the corners, where we can take $x \rightarrow \pm(a / 2-0), y \rightarrow \pm(b / 2-0)$ in the results described above. Thereby, we obtain a solution of Eqs. (20), (21), and (25) with the constants of Eqs. (26), (29), and (37).

\section{Model of Magnetic Reluctance}

\subsection{Magnetomotive Force}

We calculate the magnetomotive forces in the length direction along the surface as the following.

$$
f_{m 1}(y)=\int_{-l / 2}^{l / 2} h_{z}\left( \pm \frac{a}{2}, y, z, s\right) d z, \quad f_{m 2}(x)=\int_{-l / 2}^{l / 2} h_{z}\left(x, \pm \frac{b}{2}, z, s\right) d z
$$

Because these forces mutually differ and because they are functions of $y$ or $x$, we consider the average.

$$
f_{m}=\frac{1}{a+b}\left[\int_{-b / 2}^{b / 2} f_{m 1}(y) d y+\int_{-a / 2}^{a / 2} f_{m 2}(x) d x\right]
$$

In this case, we have the relation

$$
r_{m}(s) \equiv \frac{f_{m}}{l}=1+\left(\frac{b}{a+b}\right)^{2} \bar{f}_{m 1}+\left(\frac{a}{a+b}\right)^{2} \bar{f}_{m 2}
$$

where the following apply.

$$
\begin{aligned}
& \bar{f}_{m 1}=S_{a b}\left(\xi_{n m}\right)-S_{l}\left(\xi_{n m}\right) \\
& \bar{f}_{m 2}=S_{a b}\left(\eta_{n m}\right)-S_{l}\left(\eta_{n m}\right) \\
& S_{a b}\left(\xi_{n m}\right)=\frac{16}{\pi^{2}} \sum_{n=1}^{\infty} \sum_{m=0}^{\infty} \frac{1}{(2 m+1)^{2}} \frac{\alpha^{2}}{\xi_{n m}{ }^{2}} \\
& S_{l}\left(\xi_{n m}\right)=\frac{16}{\pi^{2}} \sum_{n=1}^{\infty} \sum_{m=0}^{\infty} \frac{1}{(2 m+1)^{2}} \frac{\alpha^{2}}{\xi_{n m}{ }^{2}} \frac{\tanh \left(\xi_{n m} l / 2\right)}{\xi_{n m} l / 2}
\end{aligned}
$$

Similar equations are also given for $S_{a b}\left(\eta_{n m}\right)$ and $S_{l}\left(\eta_{n m}\right)$. In Eqs. (42) and (43) the first term $S_{a b}(\cdot)$ is independent of the length; the second term $S_{l}(\cdot)$ has effects of the length 
(ends effect). The two terms disappear when $\alpha=0$ in the static state. Because the second term disappears when $l \rightarrow \infty$, the first term gives a model for $2 \mathrm{D}$ iron cores, where the field distribution is assumed to be uniform in the length direction. Equation (41) shows the mean magnetomotive force per unit length and describes the magnetic reluctance relative to the static reluctance, as described later.

\subsection{Magnetic Reluctance}

The magnetic reluctance is defined as

$$
R_{m}(s)=\frac{f_{m} H_{0}(s)}{\mu a b H_{0}(s)}=R_{m 0} r_{m}(s)
$$

where $R_{m 0}$ is the static reluctance given as

$$
R_{m 0}=\frac{l}{\mu a b},
$$

and $r_{m}(s)$, given by Eq. (41), is the relative value to the static reluctance.

The numerical convergence of Eqs. (44) and (45) is poor in the series of $n$ when $\alpha$ is large (at high frequency). The situation can be improved for Eq. (44). First, we note the following two different forms of the Fourier series.

$$
\begin{aligned}
& \frac{\cosh (c x)}{\cosh (c a / 2)}=\frac{\tanh (c a / 2)}{c a / 2}\left[1+\sum_{n=1}^{\infty}(-1)^{n} \frac{2 c^{2}}{c^{2}+p_{a n}{ }^{2}} \cos \left(p_{a n} x\right)\right],|x| \leq \frac{a}{2} \\
& \frac{\cosh (c y)}{\cosh (c b / 2)}=1-\frac{4}{\pi} \sum_{m=0}^{\infty} \frac{(-1)^{m}}{2 m+1} \frac{c^{2}}{c^{2}+q_{b m}{ }^{2}} \cos \left(q_{b m} y\right),|y| \leq \frac{b}{2}
\end{aligned}
$$

For $x=a / 2$ and $c=\alpha$, Eq. (48) gives

$$
\sum_{n=1}^{\infty} \frac{2 \alpha^{2}}{\alpha^{2}+p_{a n}^{2}}=\frac{\alpha a / 2}{\tanh (\alpha a / 2)}-1 \text {. }
$$

Integration of Eq. (49) between 0 and $b / 2$, and displacement of $c=\alpha$ engender

$$
\frac{8}{\pi^{2}} \sum_{m=0}^{\infty} \frac{1}{(2 m+1)^{2}} \frac{\alpha^{2}}{\alpha^{2}+q_{b m}{ }^{2}}=1-\frac{\tanh (\alpha b / 2)}{\alpha b / 2} .
$$

Incidentally, we obtain a comparative relation with Eq. (50) as

$$
\sum_{m=0}^{\infty} \frac{2 \alpha^{2}}{\alpha^{2}+q_{b m}^{2}}=\frac{\alpha b}{2} \tanh \left(\frac{\alpha b}{2}\right) .
$$

If we replace $\alpha$ by $\gamma_{b m}$, given as

$$
\gamma_{b m}^{2}=\alpha^{2}+q_{b m}^{2},
$$

and if we apply Eq. (51), then Eq. (44) is transformed to the single series shown below.

$$
\begin{aligned}
S_{a b}\left(\xi_{n m}\right) & =\frac{8}{\pi^{2}} \sum_{m=0}^{\infty} \frac{1}{(2 m+1)^{2}} \frac{\alpha^{2}}{\gamma_{b m}{ }^{2}} \sum_{n=1}^{\infty} \frac{2 \gamma_{b m}{ }^{2}}{\xi_{n m}{ }^{2}} \\
= & \frac{\alpha a}{2} \frac{8}{\pi^{2}} \sum_{m=0}^{\infty} \frac{1}{(2 m+1)^{2}} \frac{\alpha}{\gamma_{b m} \tanh \left(\gamma_{b m} a / 2\right)}+\frac{\tanh (\alpha b / 2)}{\alpha b / 2}-1
\end{aligned}
$$

Similarly, we obtain the equation

$$
S_{a b}\left(\eta_{n m}\right)=\frac{\alpha b}{2} \frac{8}{\pi^{2}} \sum_{m=0}^{\infty} \frac{1}{(2 m+1)^{2}} \frac{\alpha}{\gamma_{a m} \tanh \left(\gamma_{a m} b / 2\right)}+\frac{\tanh (\alpha a / 2)}{\alpha a / 2}-1 .
$$

In Eqs. (53) and (54), the first terms become larger with $\alpha$, and the second and third seem to be adjusting terms for small or large values of $\alpha$. When $\alpha \rightarrow 0$, Eq. (41) gives $r_{m}(0)=1$, the static value. We can check that, for $a \rightarrow \infty$, Eq. (41) coincides with the model 
of $1 \mathrm{D}$ iron core of ${ }^{(4)}$

$$
r_{m 1 b}(s)=\frac{\alpha b / 2}{\tanh (\alpha b / 2)}-\sum_{n=1}^{\infty} \frac{2 \alpha^{2}}{\beta_{b n}{ }^{2}} \frac{\tanh \left(\beta_{b n} l / 2\right)}{\beta_{b n} l / 2},
$$

where

$$
\beta_{b n}{ }^{2}=\alpha^{2}+p_{b n}{ }^{2} .
$$

\subsection{Effect of the Ends}

The part without the iron-core length in Eq. (41) is

$$
r_{m a b}(s)=1+\left(\frac{b}{a+b}\right)^{2} S_{a b}\left(\xi_{n m}\right)+\left(\frac{a}{a+b}\right)^{2} S_{a b}\left(\eta_{n m}\right) .
$$

We examine the effect of the length with the relative value shown below.

$$
\bar{r}_{m}(\omega)=\frac{r_{m}(j \omega)}{r_{m a b}(j \omega)}
$$

The data used in the computation are as follows.

$$
\mu=5000 \times 4 \pi \times 10^{-7} \mathrm{H} / \mathrm{m}, \quad \sigma=1.0 \times 10^{7} 1 / \Omega \mathrm{m}, \quad b=10 \mathrm{~mm}
$$

These data will also be used later. The numerical results will be shown with frequency $f(\mathrm{~Hz})$ instead of angular frequency $\omega(\mathrm{rad} / \mathrm{s}), \omega=2 \pi f$. The frequency is normalized as follows.

$$
\bar{f}=\frac{f}{f_{b}}, \quad f_{b}=\frac{\omega_{b}}{2 \pi}
$$

Therein, the following values are used.

$$
\begin{aligned}
& \omega_{b}=\left(1+\frac{b}{a}\right) \omega_{b 1} \\
& \omega_{b 1}=\frac{1}{\mu \sigma\left(\frac{b}{2}\right)^{2}}
\end{aligned}
$$

The angular frequency $\omega_{b 1}$ is the break frequency of the $1 \mathrm{D}$ model, the first term on the right-hand side of Eq. (55). ${ }^{(1)}$ This frequency is half that of the skin depth for the thickness of $b / 2 .{ }^{(6)}$ The frequency $\omega_{b}$ is not the break frequency of the $2 \mathrm{D}$ model, but a frequency that seems useful to display numerical results for comparison. For example, $f_{b 1}=0.101 \mathrm{~Hz}$ and $f_{b}=0.152 \mathrm{~Hz}$ for the above data and $a / b=2$.

Figure 2 portrays absolute values and arguments of Eq. (58) for $a / b=2$ with several values of $l / b$. The effects of the length are smaller for a larger length and larger for a thinner core (larger $a / b$ ); the effect is largest for the 1D iron core, whose result is also shown for $l / b=1$ with the frequency normalized by $f_{b 1}$. 

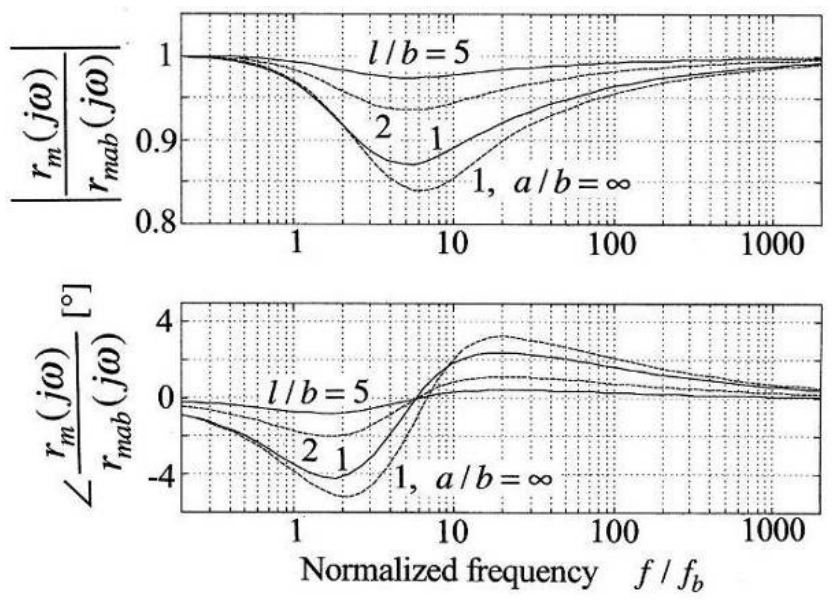

Fig. 2 Effect of the iron-core length on magnetic reluctance: Eq. (58) for $a / b=2$.
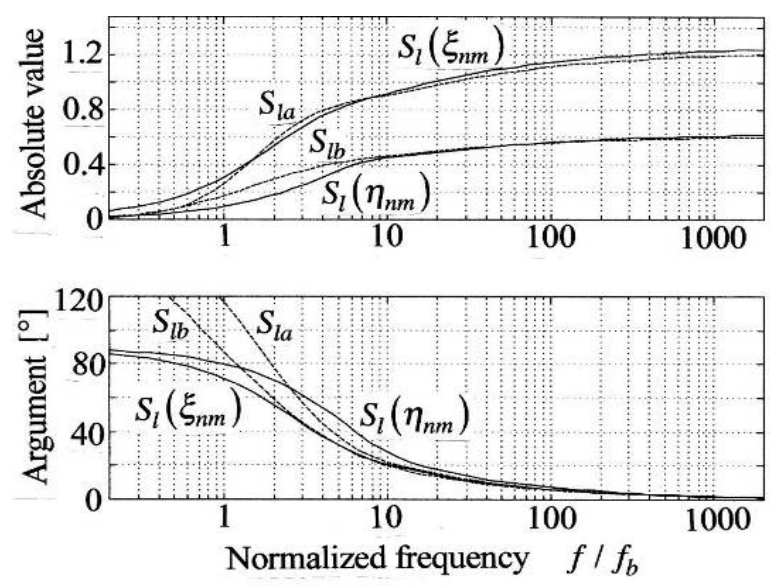

Fig. 3 Approximation of Eq. (45) by Eq. (62) for $a / b=2$ and $l / b=1$.
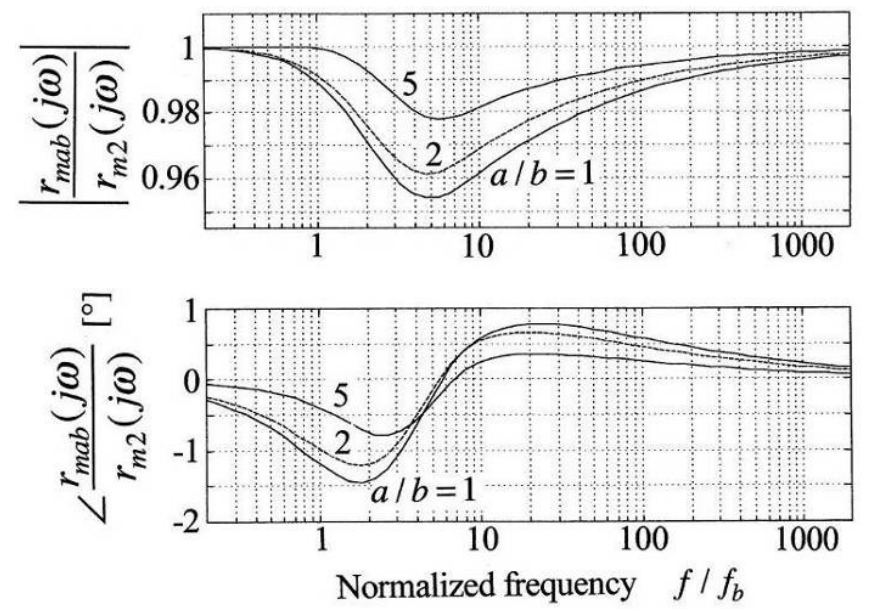

Fig. 4 Comparison of magnetic reluctance without iron-core length between 2D and 3D models: Eqs. (57) and (63). 


\section{Approximation of Series}

\subsection{Approximation of the Term with Length}

The series of Eq. (45) has a poor convergence for large $\alpha$ with $n$. Therefore, we consider the following approximation.

$$
S_{l a}=\left[\frac{\tanh (\alpha a / 2)}{\alpha a / 2}-1\right] \sum_{m=0}^{\infty} \frac{1}{(2 m+1)^{2}} \frac{\alpha^{2}}{\gamma_{b m}{ }^{2}} \frac{\tanh \left(\gamma_{b m} l / 2\right)}{\gamma_{b m} l / 2}
$$

An approximation $S_{l b}$ is written in a similar form for $S_{l}\left(\eta_{n m}\right)$. Figure 3 presents a numerical comparison for $a / b=2$ and $l / b=1$. The approximations have slightly lower absolute values at higher frequencies and a larger error at lower frequencies. The value of Eq. (45) increases monotonously with frequency and asymptotically to a real value of about $0.63(a / l)$.

\subsection{Comparison with the 2D Model}

Actually, Eq. (57) is useful for a 2D model. Another model was presented as ${ }^{(1)}$

$$
\frac{1}{r_{m 2}(s)}=\frac{\tanh (\alpha b / 2)}{\alpha b / 2}+\frac{8}{\pi^{2}} \sum_{m=0}^{\infty} \frac{1}{(2 m+1)^{2}} \frac{\alpha^{2}}{\gamma_{b m}^{2}} \frac{\tanh \left(\gamma_{b m} a / 2\right)}{\gamma_{b m} a / 2} .
$$

This model is simpler and is considered to be more reasonable as a $2 \mathrm{D}$ model because Eq. (57) was derived without the boundary condition of Eq. (9). The numerical result of their ratio, $r_{m a b}(j \omega) / r_{m 2}(j \omega)$, is portrayed in Fig. 4 for $a / b=2$ with absolute values and arguments with frequency. Actually, Eq. (57) coincides with Eq. (63) at very low and higher frequencies, but not at the intermediate frequencies. Equation (57) gives smaller values with the largest error at the normalized frequency of about 5 . The error is the largest for the square cross-section with a maximum of less than $5 \%$. The arguments are very small, less than $1.5 \mathrm{deg}$. Therefore, we can guess that the error is, at most, $5 \%$ when we neglect the boundary condition of Eq. (9). The series of Eq. (63) shows much better convergence than either Eq. (53) or (54). Therefore, we adopt Eq. (63) instead of Eq. (57).

\subsection{Comparison of Models}

Based on the discussion presented above, we write the magnetic reluctance as follows.

$$
r_{\text {map }}(s)=r_{\text {m2 }}(s)-r_{\text {mlap }}(s), \quad r_{\text {mlap }}(s)=\left(\frac{b}{a+b}\right)^{2} S_{l a}+\left(\frac{a}{a+b}\right)^{2} S_{l b}
$$

The convergence of the series $S_{l a}$ and $S_{l b}$ resembles that of $r_{m 2}(s)$. For higher frequencies, from Eqs. (41), (53), (54), and (62), we have a simple approximation of

$$
r_{m \infty}(s)=\frac{a}{a+b} \frac{\alpha b}{2}=\sqrt{\frac{1}{1+b / a} \frac{s}{\omega_{b}}} .
$$

This expression is respectively the parallel connection of two 1D models with respective heights of $a$ and $b$.

Figure 5 portrays the numerical results of Eqs. (41), (63), (64), and (65) for $a / b=2$ and $l / b=1$. Equation (64) gives a result that very closely approximates that obtained using Eq. (41). The difference between Eqs. (63) and (64) is based on the effects of the iron-core length; it is large at the normalized frequencies of $1-50$, but is smaller at the lower and higher frequencies. The difference becomes smaller as the length increases, as examined in Fig. 2 with Eq. (57) in place of Eq. (63). The approximation at higher frequencies without the length, Eq. (65), gives a good approximation at frequencies greater than about 5 . 

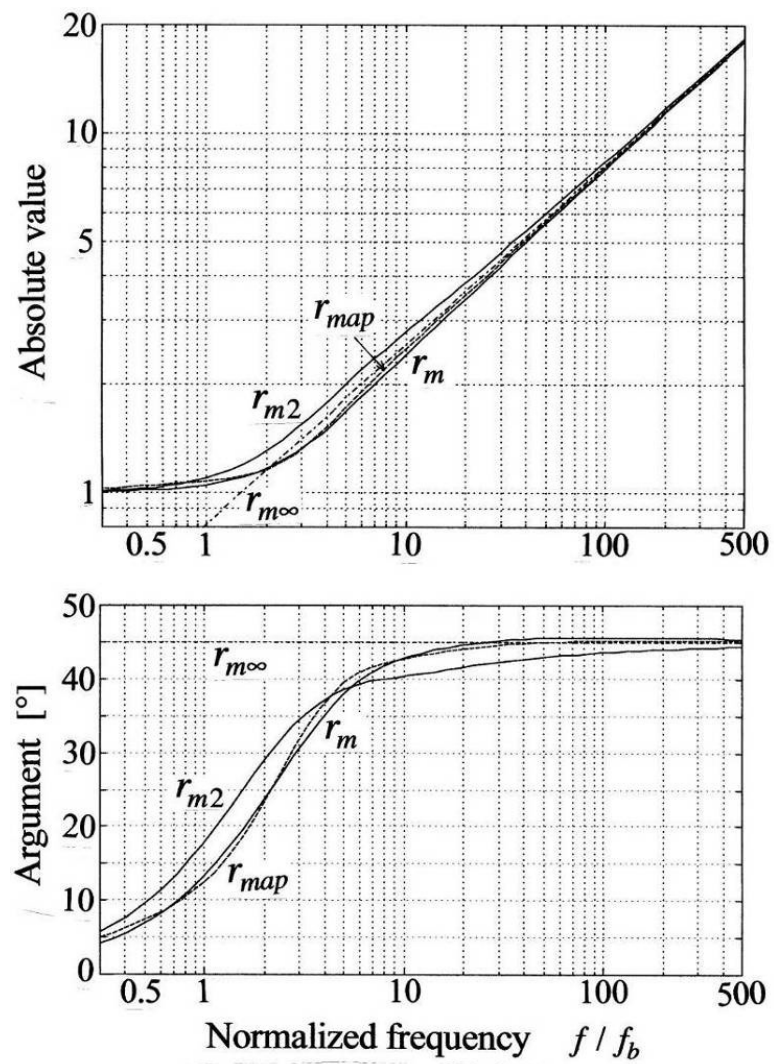

Fig. 5 Comparison of magnetic-reluctance models: Eqs. (41), (63), (64), and (65) for $a / b=2$ and $l / b=1$.

\section{Conclusions}

We tried to obtain a 3D analytical model considering the effect of the ends for the magnetic reluctance of rectangular iron cores to discuss the usefulness of the 2D model without the ends effect. We applied the theory of generalized function to the convergence of a trigonometric infinite series in considering the field distribution in the length direction. The model presented is an approximated one because we neglected the condition by which the longitudinal field should be uniform at the edge of a cross-section. The part without the ends effect, however, has a small error compared to that of the 2D model, which is considered analytically correct. Therefore, we can conclude that the approximation is very good. Approximated equations were proposed to simplify the computation. Numerical examples verified that the ends effect is smaller in the longer iron core. Consequently, we conclude that the 2D model is useful for typical cases.

\section{References}

(1) Feeley, J. J., A Simple Dynamic Model for Eddy Currents In a Magnetic Actuator, IEEE Trans. on Magnetics, Vol.32, No.2 (1996), pp.453-458.

(2) Fukata, S., A Frequency-Domain Model of Electromagnetic Actuators Composed of Solid Iron Cores, JSME Int. Journal, Vol. C-43, No. 1 (2000), pp.38-46.

(3) Frequency Characteristics of Electromagnets Composed of Solid Iron Core, Proc. of the 12th Symposium on Electromagnetics and Dynamics (in Japanese), (2000), pp. 153-156.

(4) Fukata, S., An Analysis of Frequency Characteristics of Magnetic Actuators, Trans. of JSME, Series C, Vol. 71, No. 709 (2005), pp. 2746-2753.

(5) Stoll, R.L., The Analysis of eddy currents, (1974), pp. 4-6, Clarendoon Press.

(6) Umoto, S., Electromagnetics (in Japanese), (1993), pp.17-18, p. 326, Shoukoudo. 
(7) Hsu, H.P., Fourier Analysis (in Japanese by Satou, H.), (1991), pp. 46-55, pp. 37-38, pp. 10-12, p.62, Morikita-shuppan.

(8) Schwartz, L., Mathematics for the Physical Sciences, (1966), pp. 152-153, AddisonWesley. 